\title{
Favorable Outcomes in FGFR Fusion-Positive Cholangiocarcinomas and Evolution on Treatment Noted on Circulating Tumor DNA Liquid Biopsies
}

\author{
Pashtoon Murtaza Kasi \\ Department of Hematology, Oncology and Bone Marrow Transplantation, Division of \\ Internal Medicine, University of lowa, lowa City, IA, USA
}

Keywords
Cholangiocarcinoma · Circulating tumor DNA · Liquid biopsies · FGFR · FGFR2

\begin{abstract}
Cholangiocarcinoma is a very heterogenous cancer and "target-rich" disease. While the current classifications are based on the anatomic location of these tumors (intrahepatic cholangiocarcinoma, extrahepatic cholangiocarcinoma, and gallbladder cancer), tumors within and across these disease groups have unique and often mutually exclusive molecular aberrations. Amongst these, fibroblast growth factor receptor 2 (FGFR2) fusion is one of the first amongst the list of "actionable" targets for which the US Food and Drug Administration just approved pemigatinib. This is for patients with cholangiocarcinoma who have received prior treatment and have FGFR2 fusion or another rearrangement. This was based on the results from the clinical trial FIGHT-202 (NCT02924376). At present, several FGFR inhibitors are actively being tested in several agnostic and tumor-specific clinical trials. Patients also have had the opportunity of getting access to some of these oral drugs through compassionate use programs. As a consequence, these patients have more options in addition to chemotherapy. These all tend to have "good" initial responses and improvement in performance status and later "acquired" mechanisms of resistance. The latter tend to often be gatekeeper mutations that bypass the inhibitory effects of these selective FGFR inhibitors and/or cause steric hindrance. These tumors, therefore, evolve on selective pressure (temporal heterogeneity). This can be captured noninvasively using "liquid biopsies" (circulating tumor DNA testing). Here we present cases (several years into treatment on average) showing the feasibility of using liquid biopsies (ctDNA testing) as well as the gain and later potential loss of intratumoral and tempo-
\end{abstract}


ral heterogeneity exhibited under selective pressure of these novel FGFR inhibitors, chemotherapy and/or locoregional therapies. Despite limitations in sample size and provider bias, it is important to identify these "exceptional responders" and/or better outcomes that may be inherent to the biology of FGFR fusion-positive cholangiocarcinomas.

(C) 2020 The Author(s).

Published by S. Karger AG, Basel

\section{Introduction}

Cholangiocarcinoma (CCA) is a very heterogenous cancer and "target-rich" disease (Fig. 1) [1-3]. As depicted in Figure 1, this is secondary to the increasing number of "actionable" targets for which there are either approved drugs or trials with biological rationale and/or preliminary results already showing activity. This highlights the importance and value of moving toward mandatory tumor-based somatic panel-based genetic testing to test for the presence of these targets in patients with CCA. While the current classifications are based on the anatomic location of these tumors (intrahepatic CCA [iCCA], extrahepatic CCA, and gallbladder cancer), tumors within and across these disease groups have unique and often mutually exclusive molecular aberrations. For example, while fibroblast growth factor receptor 2 (FGFR2) fusion or rearrangements and IDH1/2 mutations are often seen in iCCA, $B R A F-V 600 E$ mutations and HER2/Neu-positive tumors predominate in the subset of extrahepatic CCA and/or gallbladder cancers.

Amongst these, FGFR2 fusion is one of the first on the list of "actionable" targets for which the US Food and Drug Administration (FDA) just approved pemigatinib. This is for patients with CCA who have received prior treatment and have FGFR2 fusion or another rearrangement. This was based on the results from the clinical trial FIGHT-202 (NCT02924376) [4].

At present, several FGFR inhibitors are actively being tested in several agnostic and tumor-specific clinical trials. Patients also have had the opportunity of getting access to some of these oral drugs through compassionate use programs. As a consequence, these patients have more options in addition to chemotherapy. These all tend to have "good" initial responses and improvement in the performance status and later "acquired" mechanisms of resistance. The latter tend to often be gatekeeper mutations that bypass the inhibitory effects of these selective FGFR inhibitors or result in steric hindrance. These tumors, therefore, evolve on selective pressure (temporal heterogeneity) $[5,6]$. This can be captured noninvasively using "liquid biopsies" (circulating tumor DNA [ctDNA] testing) [5-7].

Here we present several cases of patients still undergoing active treatment showing the feasibility of using ctDNA, as well as the gain and later potential loss of intratumoral and temporal heterogeneity exhibited under selective pressure of these novel FGFR inhibitors, chemotherapy and/or locoregional therapies. Of specific note is the overall survival noted in these patients. All patients are alive and still on active treatment.

\section{Case Presentations}

The cases presented and illustrated in Figure 2 all carry the diagnosis of metastatic "intrahepatic" CCA and are still under active treatment. The specific FGFR2 fusion or rearrangement is also shown. The patients labeled case 1,2 and 3 are 52, 47 and 60 years of age, respectively. BICC1 is noted to be the FGFR2 fusion partner for 2 of these patients. The third patient has a FGFR2-LRRFIP fusion. The first 2 patients are females and 1 is male.

\section{Karger'k}




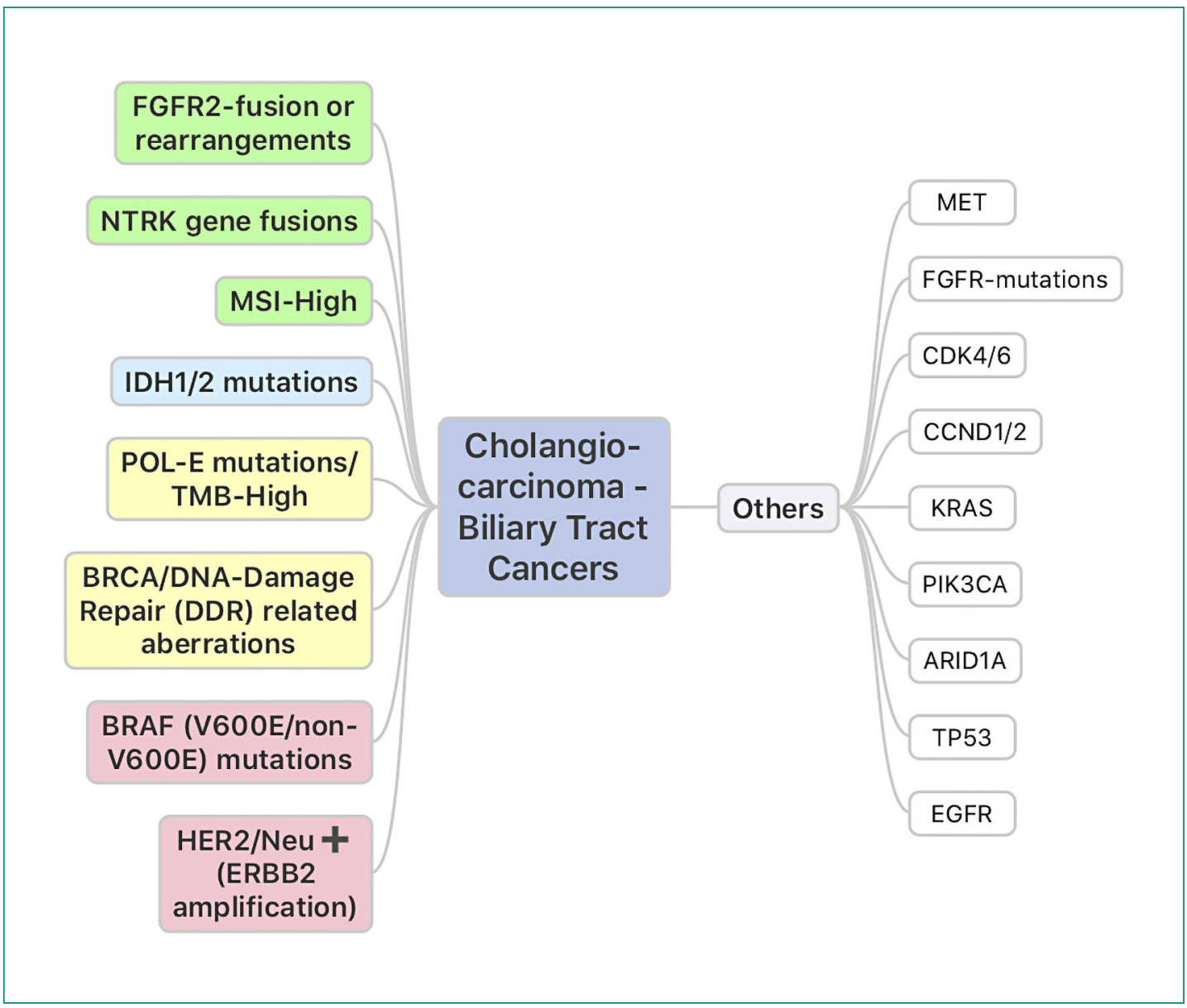

Fig. 1. Cholangiocarcinomas (biliary tract cancers) are often referred to as "target-rich." As depicted in the figure, this is secondary to the increasing number of "actionable" targets for which there are either approved drugs or trials with biological rationale and/or preliminary results already showing activity. This highlights the importance and value of moving toward mandatory tumor-based somatic panel-based genetic testing to test for the presence of these targets in patients with cholangiocarcinoma. These often tend to be mutually exclusive. While FGFR2 fusion or rearrangements and IDH1/2 mutations are often seen in intrahepatic cholangiocarcinomas, BRAF-V600E mutations and HER2/Neu-positive tumors predominate in the subset of extrahepatic cholangiocarcinomas and/or gallbladder cancers [1-3].

All the patients have had exposure to both standard of gemcitabine/platinum-based chemotherapy regimens as well as a selective FGFR inhibitor. These also include the later fluoropyrimidine/bevacizumab-based regimen for 1 patient and the addition of nab-paclitaxel to the platinum doublet for the other 2 patients.

Locoregional therapies have been employed in 2 patients and are being considered for the third. SBRT to symptomatic lung lesions in 1 patient and proton therapy to the dominant liver lesion symptomatic in another had resulted in obstructive jaundice earlier that resolved following chemotherapy and now radiation. While traditionally, locoregional treatment options including surgery are often only considered in patients without metastases, given significant intratumoral and temporal heterogeneity post-targeted therapies, this presents a unique situation where it would not be unreasonable to revisit some of these options in the right context. 


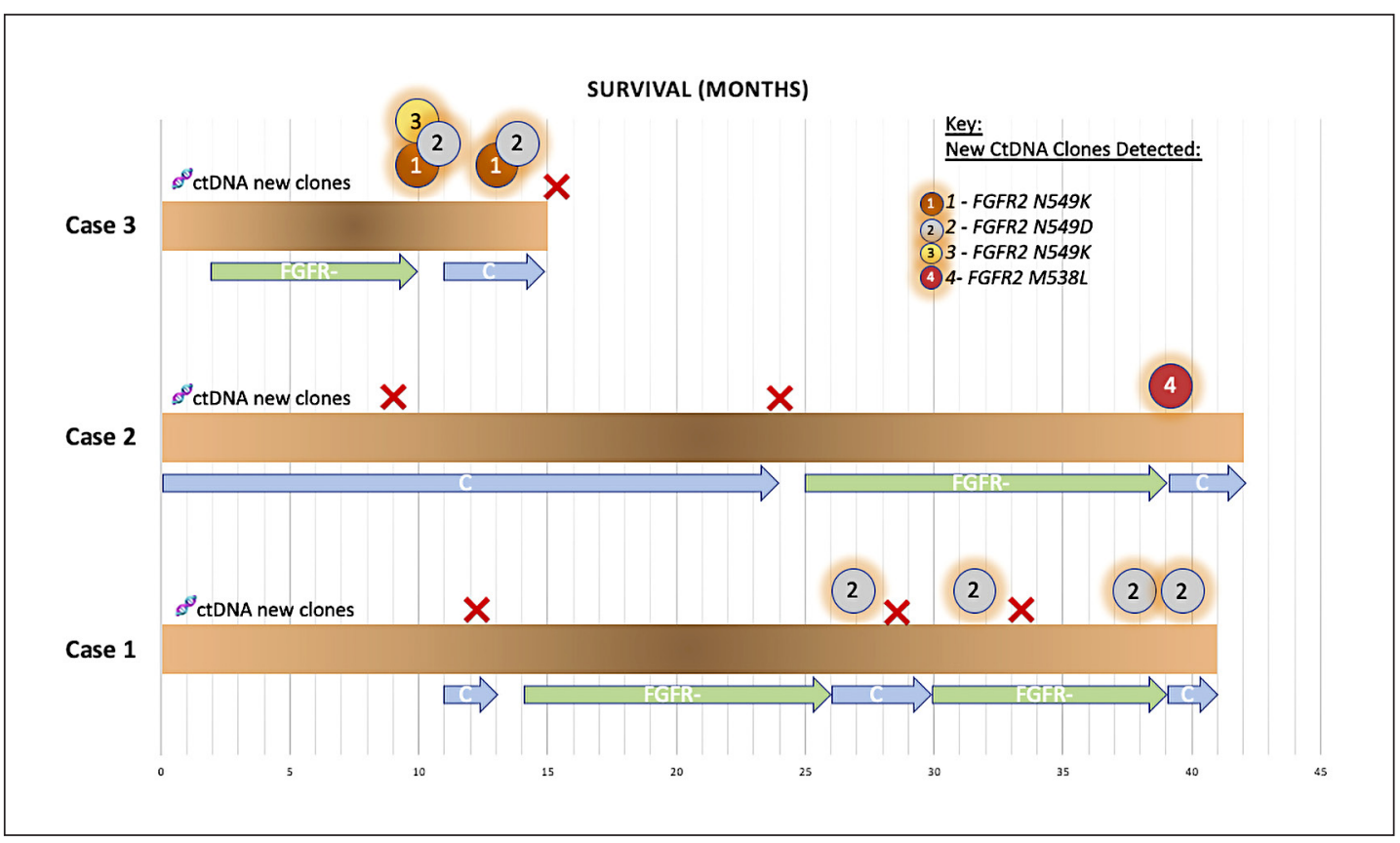

Fig. 2. Summary of outcomes and follow-up on treatment in patients with FGFR fusion-positive metastatic cholangiocarcinoma. They are all alive and on different active treatments at the time of submission of this report. The timeline is in months. As noted, patients with post-FGFR-inhibitor exposure develop clonal as well as polyclonal multiple gatekeeper mutations, some of which are known to cause activation of the pathway and/or hindrance of the FGFR inhibitor receptor binding. The circled numbers represent new clones detected on ctDNA that were not present on comprehensive NGS commercial panel-based testing platforms on their baseline tissue in all 3 and in 2 who had liquid biopsies done at baseline as well (showing that these were truly acquired). The timeline of acquisition also corroborates this since they only appeared at or before progression on FGFR inhibitors.

As noted in Figure 2, the patients' tumors following FGFR inhibitor exposure develop clonal as well as polyclonal multiple gatekeeper mutations, some of which are known to cause activation of the pathway and/or hindrance of the FGFR inhibitor receptor binding [5, 6]. The circled numbers represent new clones detected on ctDNA that were not present on comprehensive NGS commercial panel-based testing platforms on their baseline tissue in all 3 and in 2 who had liquid biopsies done at baseline as well (representing that these were truly acquired). The timeline of acquisition also corroborates this since they only appeared at or before progression on FGFR inhibitors [5, 6].

Furthermore, in studies where they had access to a repeat biopsy or tissue post-mortem, the acquired mechanisms of resistance are not present in all the tumors in the same patient at the same instant in time [5]. This supports the considerations toward employing locoregional treatments to potentially the areas that show discordant progression to "reset the clock." In patient 3 (Fig. 2), we repeated liquid biopsies following exposure to chemotherapy and FGFR treatment break. The detectable ctDNA clones disappeared. A repeat tissue biopsy also confirmed that these acquired mechanisms of resistance were either not present in the tissue sampled (intratumoral heterogeneity) or disappeared on exposure to a different drug (temporal heterogeneity). 


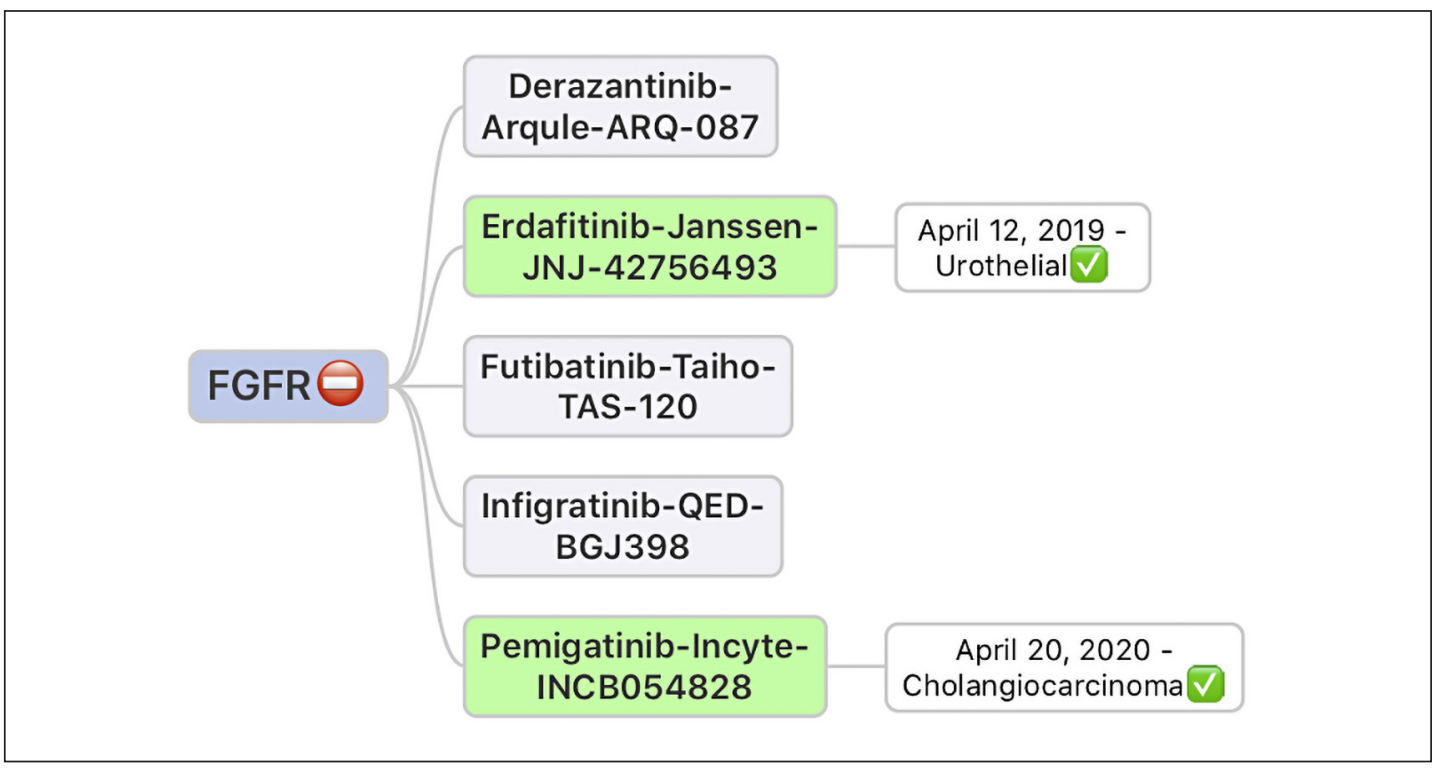

Fig. 3. Examples of just a few of the FGFR inhibitors that have already shown promise especially for FGFR fusions as opposed to mutations. The ones in green have already received US FDA approval for the disease types as shown. These drugs have variable degrees of potency and binding to various receptors/off-target effects. Resistance to one drug does not automatically exclude the use of others.

\section{Discussion and Conclusion}

FGFR fusions tend to be exclusively present in iCCA. The range reported is variable across studies. It is anywhere between 11 and 20\%; probably higher in studies that enriched/classified for iCCA better $[8,9]$.

Even within iCCA, it seems that different etiologies lead to the presence or absence of this aberration. For example, a recent study by Kongpetch et al. [9] from Thailand noted that FGFR fusions were lacking in "liver-fluke"-associated CCA as opposed to "non-fluke"-associated CCA $(1 / 121 ; 0.8 \%$; vs. $11 / 95 ; 11.6 \%, p=0.0006)$.

Previous and ongoing current classification schema and terminologies used to describe CCA are anatomic in origin. For example: iCCA, perihilar CCA, or distal CCA [10]. Other papers and trials may group and use the terminologies/abbreviations iCCA, extrahepatic CCA and gallbladder cancers. However, it is increasingly becoming clear that even within these subsets, these are very different molecular subsets of diseases. While as of right now, it is not playing too much of a role concerning the earlier stages of CCA, in the metastatic setting, the value of genomic profiling to identify these actionable findings for CCA is increasingly being recognized. While still not endorsed in guidelines, this should become standard of care at least for patients with metastatic CCA.

Trials with various FGFR inhibitors have already shown promise, especially for FGFR fusions as opposed to mutations $[8,11]$. Figure 3 shows examples of just a few of them $[8$, 11]. The ones in green have already received US FDA approval for the disease types as shown. These drugs have variable degrees of potency and binding to various receptors / off-target effects. Resistance to one drug does not automatically exclude the use of others [12].

Overall, the incidence of iCCA is rising [13]. Some of this may very well be due to appropriate diagnostic classification secondary to better imaging modalities, pathology and now 
molecular testing. A lot of these historically and even now start off as cancers of unknown primary, and then later are labeled as CCA. It is intriguing to note that the fall of numbers of cancers of unknown primary and the rise of CCA corroborate this.

In summary, CCA is indeed a "target-rich" disease [1]. Recent molecular profiling studies estimate for this to be in the range of $30-40 \%[1,9,13]$. However, it is likely even higher given the increasing number of targeted therapy options with published trials and/or close to approval/already approved. The FGFR inhibitor pemigatinib became the first targeted therapy to have disease-specific approval for the orphan disease CCA. Other approvals are likely to follow soon. While these are not curative, the case studies here illustrate the continued optimal control of this cancer. Despite metastatic disease, these patients are several years into diagnosis and still with an excellent performance status and preserved quality of life to qualify them for further treatment in trials or as part of standard of care. As these options increase, what we have learned about "continuum of care" in colorectal cancer may very well apply to CCA [14]. This infers to the fact that it is not just one thing that has changed the survival from a few months to a median of several years but the fact that it represents options that can be used one after another and later "reused/recycled/re-challenged" to extend the survival while preserving qualify of life. These observations also argue against the "treatment nihilism" often shown in these diseases whereby a significant proportion of patients are never even offered any further treatment or genomic testing. It is important to note that it is not just the targeted therapies that are options $[15,16]$. Chemotherapy combinations, for example gemcitabine, cisplatin and nab-paclitaxel, in a phase 2 trial resulted in a median overall survival of 19.2 months as opposed to the historic less than 1 year [16]. The randomized phase 3 ALLIANCE study has almost completed accrual examining the same combination.

Tumor-based (whether tissue and/or liquid where tissue is not available) genetic testing using NGS platforms should become standard of care for all metastatic CCA patients [7]. This should be endorsed by guidelines and societies so that payers would reimburse as opposed to patients/physicians being responsible for fighting denials when ordered resulting in added financial costs/anxiety. The diagnosis of cancer and its treatments already result in significant financial toxicity. Finally, the value of "repeat genomic testing" (whether tissue and preferably liquid first) merits attention following progression on targeted therapies as is illustrated in some of the recent studies [17]. It brings up an intriguing question about carefully revisiting locoregional treatments for the right patient following exposure to targeted therapies. This may allow for "revisiting/re-challenging/recycling" the same or a different inhibitor depending on the specific mechanism of resistance noted. Ongoing trials should consider and capture data on post-progression on subsequent therapies to help understand this better.

Despite limitations in sample size and provider bias, it is important to identify these "exceptional responders" and/or better outcomes that may be inherent to the biology of FGFR fusion-positive CCA.

\section{Acknowledgement}

We are deeply indebted to the patients for allowing us to present their information as a case report. Thanks are also due to the Division of Oncology and Internal Medicine at the University of Iowa for their continued support.

\section{Karger'}




\section{Case Reports in Oncology}

\begin{tabular}{l|l}
\hline Case Rep Oncol 2020;13:941-947 \\
\hline DOI: 10.1159/000509075 & $\begin{array}{l}\text { ○ 2020 The Author(s). Published by S. Karger AG, Basel } \\
\text { www.karger.com/cro }\end{array}$ \\
\hline
\end{tabular}

Kasi: ctDNA in FGFR2-Positive Cholangiocarcinomas

\section{Statement of Ethics}

The research was conducted ethically in accordance with the World Medical Association Declaration of Helsinki. The patients gave their written informed consent to present their cases as a case report.

\section{Conflict of Interest Statement}

Author P.M.K. disclosures: consultancy/advisory board: Taiho Oncology, Ipsen, Natera, Foundation Medicine; Research funding: AstraZeneca, AAA, Array Biopharma, RenovoRx.

\section{References}

1 Churi CR, Shroff R, Wang Y, Rashid A, Kang HC, Weatherly J, et al. Mutation profiling in cholangiocarcinoma: prognostic and therapeutic implications. PLoS One. 2014;9(12):e115383.

2 Lowery MA, Burris HA, 3rd, Janku F, Shroff RT, Cleary JM, Azad NS, et al. Safety and activity of ivosidenib in patients with IDH1-mutant advanced cholangiocarcinoma: a phase 1 study. Lancet Gastroenterol Hepatol. 2019 Sep;4(9):711-20.

3 Yarlagadda B, Kamatham V, Ritter A, Shahjehan F, Kasi PM. Trastuzumab and pertuzumab in circulating tumor DNA ERBB2-amplified HER2-positive refractory cholangiocarcinoma. NPJ Precis Oncol. 2019;3:19.

4 US FDA. FDA grants accelerated approval to pemigatinib for cholangiocarcinoma with an FGFR2 rearrangement or fusion. 2020. Available from: http://www.fda.gov/.

5 Goyal L, Saha SK, Liu LY, Siravegna G, Leshchiner I, Ahronian LG, et al. Polyclonal Secondary FGFR2 Mutations Drive Acquired Resistance to FGFR Inhibition in Patients with FGFR2 Fusion-Positive Cholangiocarcinoma. Cancer Discov. 2017 Mar;7(3):252-63.

6 Krook MA, Bonneville R, Chen HZ, Reeser JW, Wing MR, Martin DM, et al. Tumor heterogeneity and acquired drug resistance in FGFR2-fusion-positive cholangiocarcinoma through rapid research autopsy. Cold Spring Harb Mol Case Stud. 2019 Aug; 5(4).

7 Mody K, Kasi PM, Yang J, Surapaneni PK, Bekaii-Saab T, Ahn DH, et al. Circulating Tumor DNA Profiling of Advanced Biliary Tract Cancers. JCO Precision Oncology. 2019;(3):1-9.

8 Javle M, Lowery M, Shroff RT, Weiss KH, Springfeld C, Borad MJ, et al. Phase II Study of BGJ398 in Patients With FGFR-Altered Advanced Cholangiocarcinoma. J Clin Oncol. 2018 Jan 20;36(3):276-82.

9 Kongpetch S, Jusakul A, Lim JQ, Ng CCY, Chan JY, Rajasegaran V, et al. Lack of Targetable FGFR2 Fusions in Endemic Fluke-Associated Cholangiocarcinoma. JCO Glob Oncol. 2020 Apr;6:628-38.

10 Rizvi S, Gores GJ. Pathogenesis, diagnosis, and management of cholangiocarcinoma. Gastroenterology. 2013 Dec;145(6):1215-29.

11 Abou-Alfa GK, Sahai V, Hollebecque A, Vaccaro G, Melisi D, Al-Rajabi R, et al. Pemigatinib for previously treated, locally advanced or metastatic cholangiocarcinoma: a multicentre, open-label, phase 2 study. Lancet Oncol. 2020 May;21(5):671-84.

12 Goyal L, Shi L, Liu LY, Fece de la Cruz F, Lennerz JK, Raghavan S, et al. TAS-120 Overcomes Resistance to ATPCompetitive FGFR Inhibitors in Patients with FGFR2 Fusion-Positive Intrahepatic Cholangiocarcinoma. Cancer Discov. 2019 Aug;9(8):1064-79.

13 Chun YS, Javle M. Systemic and Adjuvant Therapies for Intrahepatic Cholangiocarcinoma. Cancer Control. 2017 Jul-Sep;24(3):1073274817729241.

14 Dekker E, Tanis PJ, Vleugels JLA, Kasi PM, Wallace MB. Colorectal cancer. Lancet. 2019 Oct 19;394(10207): 1467-80.

15 Guion-Dusserre JF, Lorgis V, Vincent J, Bengrine L, Ghiringhelli F. FOLFIRI plus bevacizumab as a second-line therapy for metastatic intrahepatic cholangiocarcinoma. World J Gastroenterol. 2015 Feb 21;21(7):2096101.

16 Shroff RT, Javle MM, Xiao L, Kaseb A0, Varadhachary GR, Wolff RA, et al. Gemcitabine, Cisplatin, and nab-Paclitaxel for the Treatment of Advanced Biliary Tract Cancers: A Phase 2 Clinical Trial. JAMA Oncol. 2019 Jun 1; 5(6):824-30.

17 Parikh AR, Mojtahed A, Schneider JL, Kanter K, Van Seventer EE, Fetter IJ, et al. Serial ctDNA Monitoring to Predict Response to Systemic Therapy in Metastatic Gastrointestinal Cancers. Clin Cancer Res. 2020 Apr 15; 26(8):1877-85. 